\title{
Effect of Adjuvant Radiotherapy in the Management of Hemangiopericytoma of the Central Nervous System:Report of 5 Cases
}

\author{
Li Chen ${ }^{1}$, Yidong Chen ${ }^{1}$, Baojin Sun ${ }^{1}$, Jing Jiang ${ }^{1}$, Feng Gao ${ }^{1}, X_{i a o g u a n g} \mathbf{Q i u}^{2}$ and Shunjiang $\mathbf{Y u}^{1 *}$ \\ ${ }^{1}$ Department of Radiation Oncology, Beijing Shijitan Hospital \& the Peking University 9th Clinical College, Beijing, 100038, P.R. China \\ ${ }^{2}$ Department of Radiation Oncology, Beijing Tiantan Hospital, the Capital Medical University, Beijing 100050, P.R.China
}

"Corresponding author: Shunjiang Yu, Department of Radiation Oncology, Beijing Shijitan Hospital \& the Peking University 9th Clinical College, 10 Tieyiroad, Yangfangdian, Haidian District, Beijing, 100038, P.R. China, Tel: (86)10- 6392 6203; Fax: (86) 10- 6392 6603; E-mail: yushunjiang@yahoo.com

Received date: Aug 12, 2013, Accepted date: Jul 22, 2014, Publication date: Jul 31, 2014

Copyright: (c) 2014 Chen L, et al. This is an open-access article distributed under the terms of the Creative Commons Attribution License, which permits unrestricted use, distribution, and reproduction in any medium, provided the original author and source are credited.

\begin{abstract}
Objective: Primary intracranial or spinal hemangiopericytoma (HPC) represents a rare tumour that is more difficult to distinguish from other CNS tumours based on clinical symptoms and imaging findings. In this article, here we present 5 cases with HPC ( 3 intracranial and 2 spinal) with histologically confirmed were treated at our hospital between June 2009 to January 2010, and a review of the literature pertaining to the diagnosis, clinical management, and so on.
\end{abstract}

Methods: Clinical data of the 5 patients with HPCs from cerebra and spinal cord including the manifestations of imaging, pathology, treatment and prognosis factors were investigated, and relevant literatures were reviewed. Of which, 3 males, 2 females, age ranged from 6-35 years old, median age at primary diagnosis was 29 years.

Results: 3 patients underwent craniotomy and the other 2 patients underwent spinal surgery. Total mass removal was achieved in 1 case and subtotal removal in 4 cases. All 5 patients received radiotherapy after resection, A total dose of $59.4 \mathrm{~Gy} / 33 \mathrm{f}$ was delivered in a fractionation of $5 \times 1.8 \mathrm{~Gy}$ per week for the 3 patients with cerebral lesions using three dimensional conformal radiation therapy (3D-CRT) or intensive modulated radiation therapy (IMRT) technique, and $45 \mathrm{~Gy}$ in 25 fractions for the 2 patients with spinal lesions. The patients were followed up varied from 6 to 27 months, and none of the patients was found with evidence of radiation complications. Acute toxicity was mild including skin erythema and alopecia. Follow-up of the patients has not yet discovered that a tumour recurrence and extra-cranial metastasis all remain alive up to date.

Conclusions: Multidisciplinary care should be highly advocated in the management of intracranial or spinal HPC. Surgical resection, either complete or subtotal excision, followed by postoperative radiotherapy, will provide the patients with higher probability for disease-free survival.

Keywords: Hemangiopericytoma; Radiotherapy; Surgery; Intracranial; Spinal
Abbreviations
3D-CRT: Three Dimensional Conformal Radiation Therapy; IMRT: Intensive Modulated Radiation Therapy

\section{Introduction}
Hemangiopericytoma (HPC, or HAP) of the central nervous system (CNS) is a rare vascularized mesenchymal tumour that originate from pericytes, the contractile cells surrounding capillaries, and develops from malignant transformation of pericytes, it can occur anywhere in the human body because of the ubiquity of pericytes in all mesenchymal tissues, especially in bone, kidney, liver, skin, and central nervous system [1]. Intracranial HPC accounts for $2 \% \sim 4 \%$ of meningeal tumour, and it remains a rare entity and has been estimated to represent $0.4 \%$ of all primary central nervous system tumours, there is no significant difference of the HPC incidence in gender, and ages of around 40-50 are most commonly affected, the natural course of the disease can be very long. The clinical manifestations usually are

dizziness, diplopia, and so on. Pathologically, HPC of the CNS is mostly lobulated solid tumours with clear boundaries. Microscopic examination typically reveals highly cellular tumours, with round or fusiform tumour cells demonstrating ill-defined cytoplasm, oval nuclei, and small prominent nucleoli, and more common nuclear fission $[2,3]$. The immunohistochemical profile of HPC of the CNS differs from that of meningioma and thus is imperative in securing the diagnosis. Whereas both meningiomas and HPCs are often vimentin positive, reflecting their mesenchymal origin. As tumours grow quickly, anaplastic HPC is typically characterized by the presence of necrosis and cystic changes, and the tumour is abundant in blood vessels, and the vessel wall is thinner, the lumen is coating unique by a layer of flat endothelial cells lining. There were non-specific antibodies, but the expression of Vimentin may positive $(++)$, indicate that HPC cells may arise from mesenchyma. Radiographically, intracranial HPCs are difficult to differentiate from meningiomas, schwannomas, and solitary fibrous tumours. When the tumour is small, imaging often misdiagnosed as meningioma. MRI examination of these tumours is typically isointense with cortical gray matter on T1WI and isointense to mildly hyper-intense on T2WI. Contrast enhancement images typically demonstrate intensely enhancing extraaxial lesions, often with a discernable dural attachment. Some authors 
have suggested that the imaging characteristics may help to distinguish HPC from meningioma. Well-defined lytic destruction of adjacent skull on CT scan can help the HPC diagnosis; the reliable radiographic characteristic differentiating HPC from meningioma is usually the presence of calcification within the lesion, which effectively excludes HPC from the differential diagnosis $[4,5]$.

HPCs are highly vascularized tumours, and the cells of the intracranial hemangiopericytoma comprise the walls of capillaries and postcapillary venules. The clinical, radiographic, and gross characteristics of these tumours often are indistinguishable from meningiomas, and sometimes easily misdiagnosed as meningioma or hemangioma [2-4]. Because HPC closely mimics meningioma in clinical and radiographic presentation, histological confirmation is the only definitive means of distinguishing the diagnosis. In addition, despite sharing a common arachnoid location with benign meningioma, HPC is distinguished by a more aggressive biology that facilitates local recurrence and distant metastasis, they are mesenchymal neoplasms that grow rapidly; the tumours had distant metastasis and invasive ability, and can be throughout the body. Therefore, early diagnosis and effective treatment are important for local control. Intracranial HPC has a high tendency to recur locally and is associated with a high risk of metastasizing. Because HPC is difficult to cure with surgery, postoperative radiotherapy (RT) may play an important role in the treatment of HPC. The role of precision radio-surgery has been investigated in the treatment of smaller HPCs, and appears to hold some promise for maintaining local control of tumour growth, a stereotactic Gamma Knife radiosurgery has been shown to be very effective in controlling HPC both inside and outside the central nervous system, with fractionated precision radiation therapy, high doses of radiation can be applied to a defined target while sparing normal tissue structures such as optic nerves, chiasm, and the spinal cord [6-8]. Here we report our experience from June 2009 to January 2010 in treating five patients with intracranial HPC using radiotherapy after tumour surgical resection, and evaluate the role of three dimensional conformal radiation therapy (3D-CRT) and intensive modulated radiation therapy (IMRT) in the management of the HPC of the CNS, and offer an analysis of treatment outcomes based on our experience.

\section{Materials and Methods}

\section{General materials}

Five patients with histological confirmed HPC were treated at our hospital between June 2009 to January 2010. The tumours were localized respectively within the cerebellum, temporal-parietal, frontal-temporal regions $(n=3)$, and spine T4-T8, L3-S2 regions $(n=2)$. Of which, 3 males, 2 females, age ranged from 6-35 years old, median age at primary diagnosis was 29 years. Median disease course was 16 months (range, 1-36months). The patients presented with neurological symptoms, including headache, vision damage, nausea, vomiting, and motor deficits. After primary diagnosis, surgical resection was performed in all 5 patients (table 1).

\begin{tabular}{|c|c|c|c|c|c|c|c|}
\hline Patients & Gender & Age & Lesion location & $\begin{array}{l}\text { Histo-Pathology } \\
\text { confirmed }\end{array}$ & $\begin{array}{l}\text { Surgical } \\
\text { pattern }\end{array}$ & $\begin{array}{l}\text { Radiation } \\
\text { Dose delivered }\end{array}$ & $\begin{array}{l}\text { Serious Side- } \\
\text { effects }\end{array}$ \\
\hline No.01 & M & 6 & cerebellum & HPC & Total gross & $59.4 \mathrm{~Gy} / 1.8 \times 33 \mathrm{f}$ & No \\
\hline No.02 & $\mathrm{F}$ & 29 & temporal- apical & $\mathrm{HPC}$ & subtotal & $59.4 \mathrm{~Gy} / 1.8 \times 33 f$ & No \\
\hline No.03 & M & 25 & frontal- temporal & $\mathrm{HPC}$ & subtotal & $59.4 \mathrm{~Gy} / 1.8 \times 33 \mathrm{f}$ & No \\
\hline No.04 & M & 26 & T4-T8 & $\mathrm{HPC}$ & subtotal & 45 Gy/1.8x25f & No \\
\hline No.05 & $\mathrm{F}$ & 35 & L3-S2 & HPC & subtotal & $45 \mathrm{~Gy} / 1.8 x 25 f$ & No \\
\hline
\end{tabular}

Table 1: General characteristics of five patient's with HPC and clinical managements.

\section{Clinical and pathological features}

It mainly manifested symptoms with a medical history of tumour pressing tissue results in local symptoms. Of these, it has headache in 3 cases, decreased vision and visual field defect in 1 case, and the side limb muscle strength weakened in 2 cases, the side ptosis in 1 case. Preoperatively, all 5 patients underwent enhanced MRI examination, MRI examination showed T1 equal signal, T2 slightly higher signal, with significantly contrast enhance, there are sometimes cystic changes or tumour focal necrosis in larger tumours, invasion of the tumour to the surrounding tissue may have edema around the tumour (Figure1).
Exposure of the lesion revealed it to be a well-encapsulated, gray, fibrous lesion. Metastatic workup including CT of the chest, abdomen, and pelvis didn't reveal any evidence of extra cranial-spinal HPC. Histological tissue sections of the tumours generally revealed a neoplasm of high cellularity composed of cells with oval to spindled nuclei in a densely hyalinized background and scattered "staghorn" ectatic blood vessels, a reticulin stain highlighted abundant pericytic and vascular collagen. Immunohistochemistry was carried out using antibodies to factor CD34, EMA, vimentin, and S-100. The tumour showed immunoreactivity with antibodies to vimentin, CD-34. Collectively, the microscopic features and immunohistochemical profile confirmed the diagnosis of HPC (Figure 2). 
Citation: Chen L, Chen Y, Sun B, Jiang J, Gao F, et al. (2014) Effect of Adjuvant Radiotherapy in the Management of Hemangiopericytoma of the Central Nervous System:Report of 5 Cases. J Nucl Med Radiat Ther 5: 184. doi:10.4172/2155-9619.1000184

Page 3 of 6

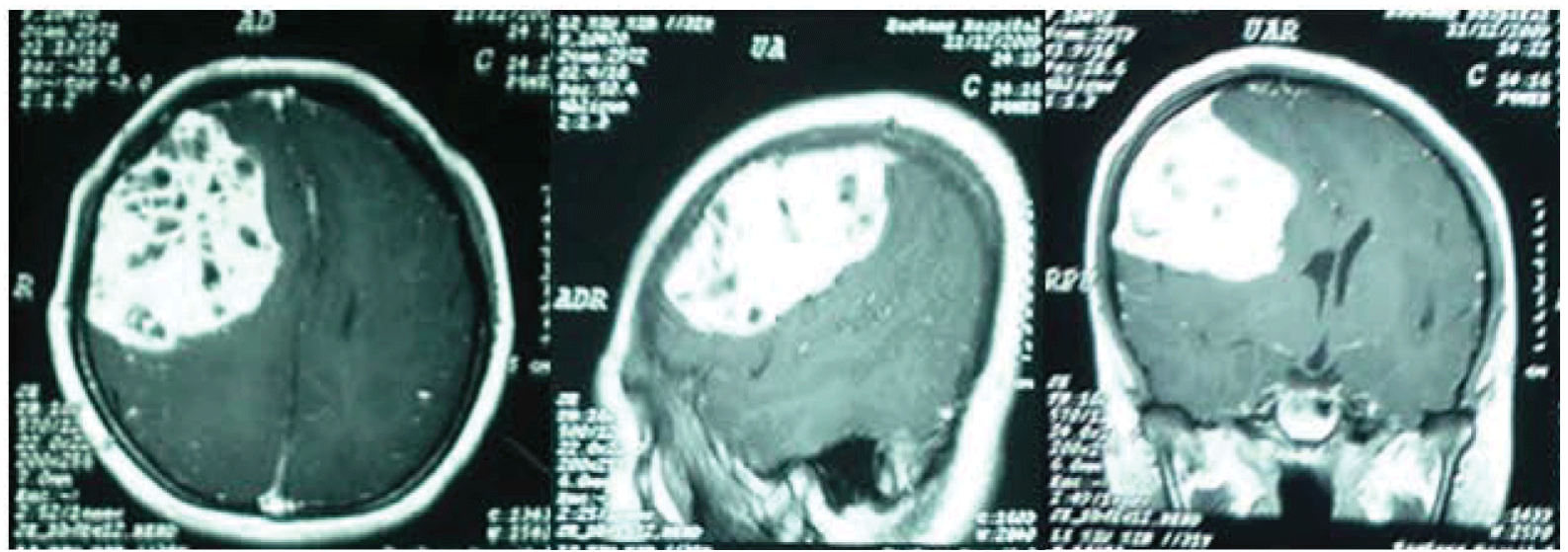

Figure 1: Cerebral MRI features of contrast-enhancing of intracranial HPC before Surgery. Contrast MRI of the head demonstrated a huge enhancing mass of right temporal- apical lesion in the axial, sagittal, and coronal views.

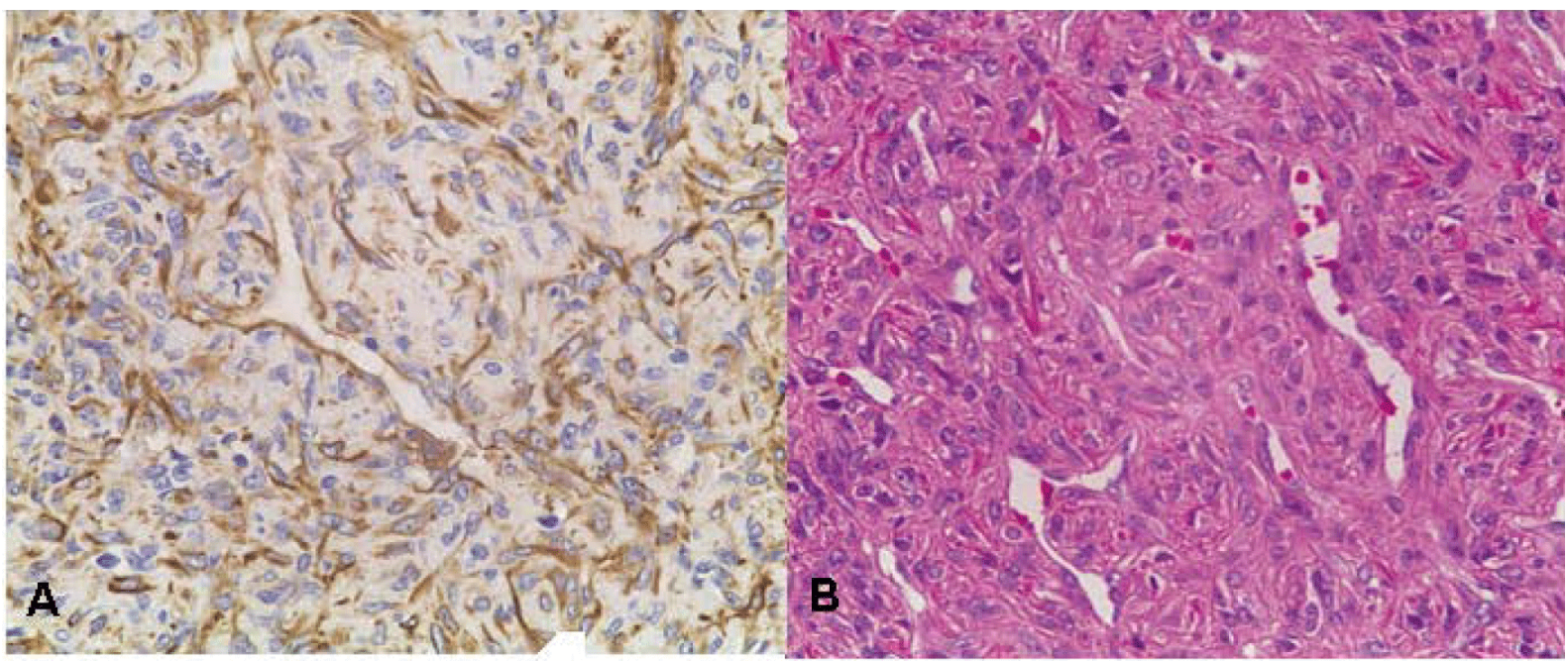

Figure 2: Pathological manifestations of cranial HPC (x200). A: a staining IHC, vimentin-positive. B: HE staining tumour cells, round or irregular shape, occasionally arranged in turbine-like, nuclear fission or nuclear shaped nuclei is more common, tumour mass is rich in blood vessels, and blood vessel walls thin. Lumen are only coated with a thin layer of the flat endothelial cells, and some abnormal blood vessels characterized with expansion.

\section{Treatment options}

Neurologic examination was intact, with diminished temperature perception of hand and foot in 2 patients with spinal lesions. After primary diagnosis, a surgical resection was performed in all 5 patients either completes or subtotal resection, the surgical patterns depend mainly on lesion's location, and the patients did not demonstrate any new neurologic deficits. All 5 patients received postoperative MRI examination to confirm a gross total or subtotal resection. Postoperatively, 5 patients did not demonstrate any new neurologic deficit. Radiotherapy for patients with microscopic residual disease or with resected cerebral or spinal involvement is planned as follows. The patient received postoperatively radiation therapy by $3 \mathrm{D}-\mathrm{CRT}$ or IMRT technique consisting of $59.4 \mathrm{~Gy}$ in $1.8 \mathrm{~Gy}$ per fraction in total 33 fractions in continuous irradiation using Linac with $6 \mathrm{MV}-\mathrm{X}$ rays (Varian $21 \mathrm{EX)} \mathrm{for} \mathrm{intracranial} \mathrm{lesons,} \mathrm{and} \mathrm{45Gy} \mathrm{in} \mathrm{1.8Gy} \mathrm{per} \mathrm{fraction}$ for the spinal lesions. The area to be irradiated is determined by correlating preoperative imaging studies, intraoperative findings, and the pathological review of the type. The target volume was generally defined as the tumour bed. For macroscopic residual tumours, the planning target volume (PTV) included the area of contrast enhancement adding a safety margin of $10-20 \mathrm{~mm}$ for typical microscopic spread, depending on the location of the tumour and on histology. The 95\% PTV isodose line encompassed the target volume. All patients were seen for regular follow-up visits at least 6 to 12 months after completion of radiotherapy. Follow-up examinations included complete clinical examinations with a thorough neurological assessment and contrast- enhanced MRI will be continuously followed. The treatment planning showed as in Figures 3,4 and 5. 
Citation: Chen L, Chen Y, Sun B, Jiang J, Gao F, et al. (2014) Effect of Adjuvant Radiotherapy in the Management of Hemangiopericytoma of the Central Nervous System:Report of 5 Cases. J Nucl Med Radiat Ther 5: 184. doi:10.4172/2155-9619.1000184

Page 4 of 6

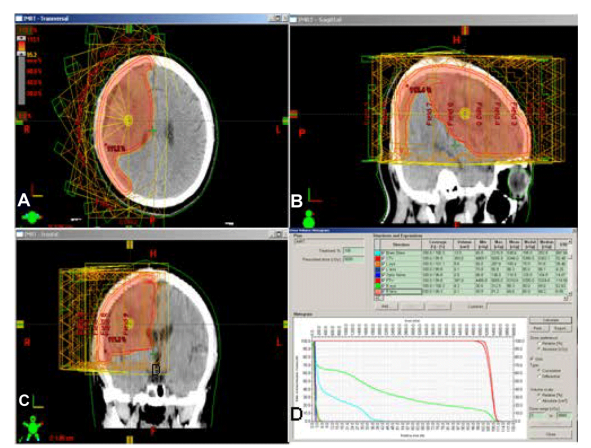

Figure 3: Typical treatment plan for IMRT technique of Hemangiopericytoma of the right temporal- apical cerebra in (A) axial view, (B) sagittal view, (C) coronal view, and (D) dose-volume histogram (DVH). The patient received postoperatively radiation therapy by IMRT technique consisting of $59.4 \mathrm{~Gy}$ in 1.8 Gy per fraction in total 33 fractions in continuous irradiation using Linac with $6 \mathrm{MV}-\mathrm{X}$ rays (Varian $21 \mathrm{EX}$ ). The area to be irradiated is determined by correlating preoperative imaging studies, intraoperative findings, and the pathological review of the type. The target volume was generally defined as the tumour bed. For macroscopic residual tumours, the planning target volume (PTV) included the area of contrast enhancement adding a safety margin of $10-20 \mathrm{~mm}$. The $95 \%$ PTV isodose line encompassed the target volume.

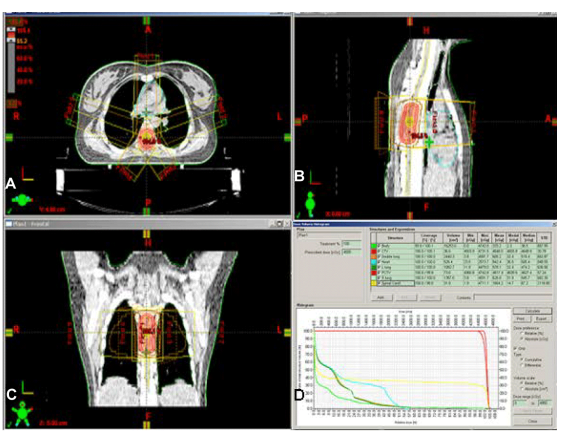

Figure 4: Typical treatment plan for 3D-CRT for HPC of the upper spine lesion (T4-T8) in (A) axial view, (B) sagittal view, (C) coronal view, and (D) dose-volume histogram (DVH). The patient received postoperatively radiation therapy by $3 \mathrm{D}$-CRT technique consisting of $45 \mathrm{~Gy}$ in $1.8 \mathrm{~Gy}$ per fraction in total 25 fractions in continuous irradiation using Linac with $6 \mathrm{MV}-\mathrm{X}$ rays (Varian $21 \mathrm{EX}$ ). The area to be irradiated is determined by correlating preoperative imaging studies, intraoperative findings, and the pathological review of the type. The target volume was generally defined as the tumour bed. For macroscopic residual tumours, the planning target volume (PTV) included the area of contrast enhancement adding a safety margin of $10 \mathrm{~mm}$. The 95\% PTV isodose line encompassed the target volume.

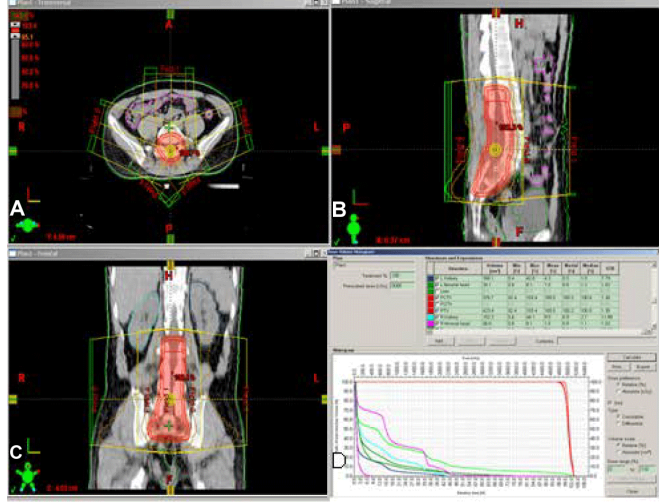

Figure 5: Typical treatment plan for 3D-CRT for HPC of the low spine(L3-S2) in (A) axial view, (B) sagittal view, (C) coronal view, and (D) dose-volume histogram (DVH). The patient received postoperatively radiation therapy by $3 \mathrm{D}-\mathrm{CRT}$ technique consisting of $45 \mathrm{~Gy}$ in $1.8 \mathrm{~Gy}$ per fraction in total 25 fractions in continuous irradiation using Linac with $6 \mathrm{MV}-\mathrm{X}$ rays (Varian $21 \mathrm{EX}$ ). The area to be irradiated is determined by correlating preoperative imaging studies, intraoperative findings, and the pathological review of the type. The target volume was generally defined as the tumour bed. For macroscopic residual tumours, the planning target volume (PTV) included the area of contrast enhancement adding a safety margin of $10 \mathrm{~mm}$. The $95 \% \mathrm{PTV}$ isodose line encompassed the target volume.

\section{Results}

Total removal was achieved in 1 case and subtotal removal was achieved in 4 cases. All 5 patients recovered well after operation, and previous symptoms were relieved mostly, and no complications occurred. All 5 patients received postoperative radiotherapy. Radiotherapy was well tolerated by all patients and could be completed without interruptions. Acute toxicity by radiotherapy was mild including skin erythema and alopecia, as evaluated with common toxicity criteria (CTC version 3.0). The patients were followed up for 6 to 27 months, and all remain alive during follow-up. No recurrence or metastasis was found in the 5 cases. To date, no severe late side-effects have been observed.

\section{Discussion}

Hemangiopericytoma in the central nervous system (CNS) are extremely rare, and it represents less than $0.5 \%$ of all intracranial tumours [9-11]. Intracranial hemangiopericytomas usually present as solitary, supratentorial, dural-based lesions, and often arising from the falx, tentorium, sagittal sinuses in the vicinity and skull base. Their relative abundant vascularity coupled frequently with their association with vital neural structures. In addition, tumour's adhesions to the surrounding tissue often occurs [7]. Common clinical symptoms are headache, and gradual emergence signs of increased brain pressure relying on tumour location and tumour growth, sometimes with acute intracranial hemorrhage as the main clinical manifestations, there are more than half of the patients with intracranial HPC appeared increased cranial pressure at initial diagnosis $[12,13]$. When the tumour grows to a certain extent, the mass shape manifests more 
irregular, often lobulated, which reflects tumour invasive growth extent. Intracranial HPC is rich with blood supply; hemorrhage and necrosis often appear with liquefaction and cystic changes, so its MRI signal and tumour entities of MRI enhancing showed significant enhancement effect of tumour heterogeneity. The initial diagnosis of these five patients with HPC in our study is typically made after careful review of the slides, as the histological characterization is essential for treatment decisions and prognosis.

Currently, surgical resection either complete, subtotal, or gross removal is the main way in the management of HPC, complete resection of the tumour at the time of initial surgery should be attempted. However, surgical intra-operative bleeding is often much during resection due to the high vascularity, which may lead to high surgery related morbidity and mortality, and in this case, it can be a great difficulty to undergo total tumour removal. A study by Soyuer showed that the 5-year local control rates after surgery were $84 \%$ after gross total resection and $38 \%$ after partial resection [8], hence extent of removal should be as great as extensive possible. When safe and feasible, gross total resection should be pursued as an initial surgical strategy to maximize overall survival. Given the rarity and aggressive nature of the HPCs, there is presently in the field no common understanding reached on standard care of the HPC to date, but most investigators believe that the standardized method of treatment should include gross total resection, followed by postoperative radiotherapy, and many studies have shown a benefit of combination of surgical resection and postoperative radiotherapy in local control both for gross residue and microscopic incomplete excision in the management of the HPC, and most of them suggested that radiotherapy after surgical resection should be routinely given [6,14-18]. In our study, all these 5 patients with HPC received postoperative radiotherapy, the dose delivered was 59.4Gy for the intracranial HPC, and 45Gy for the spinal HPC. There are no conclusive dose was recommended currently for HPC, it was reported a median total dose of 54 Gy was delivered in a routine fractionation, the overall survival rates at 5 and 10 years are $100 \%$ and $64 \%$, respectively [6]. It's reported that the response of HPCs to radiotherapy is dose dependent, with overall dose of $45 \mathrm{~Gy}$ or higher resulting in superior local control $[19,20]$. The role of chemotherapy in the treatment of systemic HPC is unclear; however, some patients have demonstrated modest benefit from chemotherapeutic regimens [21-23]. A study suggested that chemotherapy has a positive effect for patients with recurrence of HPC, but large sample study and long-term observation should be carried out [24]. In addition to their propensity for local recurrence, HPCs also metastasize outside the CNS, it's reported that the average time to local recurrence is from 12 to 47 months, and the average time to development of extra-cranial metastases ranges from 63 to 99 months after initial surgical resection, and the most common sites of distant metastases are bone, liver, lung, and the skeletal muscle, but early systemic metastasis is very rare $[19,25,26]$. Radiotherapy shows promise in preventing local tumour progression, but recurrence sometimes still remains a common treatment outcome, adjuvant postoperative radiation is an attractive option for surgical remaining tumour $[6,7,15,16,18,26-28]$. There was a trend toward prolonged time to recurrence among patients who received adjuvant radiation in addition to their primary surgical resection. More specifically, this trend was seen among patients receiving subtotal resection plus adjuvant radiation compared with gross resection alone [15]. In our study, it has not yet discovered a tumour recurrence and extra-cranial and extra-spinal metastasis during the follow-up period; all patients remain alive to date. As most of cases tend to locally recur by many years after treatment with gross total resection and adjuvant RT, hence it is advocated that long-term follow-up should be carried out. Recent clinical study series have reported rates of HPC recurrence around $90 \%$ as early as 7 months after the time of initial treatment, and the mean overall survival rates for HPC have been reported to be as low as 7 years after diagnosis. The poor prognosis associated with intracranial HPC has led investigators to advocate aggressive treatment combining radical surgical resection with adjuvant radiotherapy $[15,16]$.

Although most authors recommend at present a multimodality treatment strategy, the overall literature regarding intracranial HPC remains very limited, especially concerning clinical sample size and long-term follow-up of patients. In a previous study in patients with intracranial HPC in the published literature, it was found that gross total resection (GTR) provided the greatest survival advantage and that the addition of postoperative adjuvant radiation did not seem to confer a survival benefit but can extend the disease-free interval $[17,18]$. Metastasis remains a potential consequence of HPC progression, but our data can't suggest an optimal strategy as the cases are very limited. Given literature that radiation does not halt the development of metastasis, we believe that it is prudent to maintain surveillance of all patients with HPC of the CNS after treatment, regardless of extent of resection or adjuvant radiation. The lack of a standard of care for HPC patients makes it practically important to perform a complete and aggressive workup, especially among patients presenting with recurrent HPC.

\section{Conclusion}

Intracranial and spinal HPCs are rare, and often difficult to differ from meningiomas based on radio-imaging and characteristics. The biological behavior of the HPC is more tendency to recur locally and to metastasize extra-spinocranially, complete or gross total resection at the initial time are highly advocated. Postoperative radiotherapy is a triable method to reduce local recurrence for CNS patients with HPCs, radiotherapy should be applied regardless of the surgical residue extent. Long-term follow-up of these patients is necessary, as the recurrence often takes long time to develop.

\section{Acknowledgments}

We would like to thank Dr. L. Luo for his contribution in preparing pathological report for this publication.

\section{References}

1. Enzinger FM, Smith BH (1976) Hemangiopericytoma. An analysis of 106 cases. Hum Pathol 7: 61-82.

2. Tsou H, Wang Y, Wei S, Yang D (2002) Intra-extracranial hemangiopericytoma: Clinical manifestations, histopathological features, diagnosis, treatment, and outcomes. Chin Med J 65: 314-319.

3. Guthrie BL, Ebersold MJ, Scheithauer BW, Shaw EG (1989) Meningeal hemangiopericytoma: histopathological features, treatment, and longterm follow-up of 44 cases. Neurosurgery 25: 514-522.

4. Huang TY (2002) Intracranial hemangiopericytoma: diagnosis, treatment and outcome. Zhonghua Yi Xue Za Zhi (Taipei) 65: 305-306.

5. Kumar R, Wani AA (2006) Unusual tumors of the posterior fossa skull base. Skull Base 16: 75-84.

6. Combs SE, Thilmann C, Debus J, Schulz-Ertner D (2005) Precision radiotherapy for hemangiopericytomas of the central nervous system. Cancer 104: 2457-2465.

7. Dufour H, Métellus P, Fuentes S, Murracciole X, Régis J, et al. (2001) Meningeal hemangiopericytoma: a retrospective study of 21 patients with 
Citation: Chen L, Chen Y, Sun B, Jiang J, Gao F, et al. (2014) Effect of Adjuvant Radiotherapy in the Management of Hemangiopericytoma of the Central Nervous System:Report of 5 Cases. J Nucl Med Radiat Ther 5: 184. doi:10.4172/2155-9619.1000184

Page 6 of 6

special review of postoperative external radiotherapy. Neurosurgery 48 756-762.

8. Soyuer S, Chang EL, Selek U, McCutcheon IE, Maor MH (2009) Intracranial meningeal hemangiopericytoma: the role of radiotherapy: report of 29 cases and review of the literature.Cancer 100: 1491-1497.

9. Jääskeläinen J, Servo A, Haltia M, Wahlström T, Valtonen S (1985) Intracranial hemangiopericytoma: radiology, surgery, radiotherapy, and outcome in 21 patients. Surg Neurol 23: 227-236.

10. Wu W, Shi JX, Cheng HL, Wang HD, Hang $\mathrm{CH}$, et al. (2009) Hemangiopericytomas in the central nervous system. J Clin Neurosci 16: 519-523.

11. Mekni A, Kourda J, Chelly I, Ferchichi L, Bellil K, et al. (2008) Hemangiopericytoma in the central nervous system. A study of eight cases. Neurochirurgie 54: 15-20.

12. Kaen A, Arrese I, Lagares A, Cabello A, Lobato RD (2007) Haemangiopericytoma presenting with acute intracerebral haemorrhage. Acta Neurochir (Wien) 149: 415-418.

13. Ajani OA1, Ejeckam GC, Raza A (2003) Haemangiopericytoma presenting as acute subdural haematoma. Br J Neurosurg 17: 559-561.

14. Kano H, Niranjan A, Kondziolka D, Flickinger JC, Lunsford LD (2008) Adjuvant stereotactic radiosurgery after resection of intracranial hemangiopericytomas. Int J Radiat Oncol Biol Phys 72: 1333-1339.

15. Rutkowski MJ, Jian BJ, Bloch O, Chen C, Sughrue ME, et al. (2012) Intracranial hemangiopericytoma: clinical experience and treatment considerations in a modern series of 40 adult patients. Cancer118: 1628-1636.

16. Ghia AJ, Allen PK, Mahajan A, Penas-Prado M, McCutcheon IE, et al. (2013) Intracranial hemangiopericytoma and the role of radiation therapy: a population based analysis. Neurosurgery 72: 203-209.

17. Schiariti M, Goetz P, El-Maghraby H, Tailor J, Kitchen N (2011) Hemangiopericytoma: long-term outcome revisited. Clinical article. J Neurosurg 114: 747-755.
18. Rutkowski MJ, Sughrue ME, Kane AJ, Aranda D, Mills SA, et al. (2010) Predictors of mortality following treatment of intracranial hemangiopericytoma. J Neurosurg 113: 333-339.

19. Kim JW, Kim DG, Chung HT, Paek SH, Kim YH, et al. (2010) Gamma Knife stereotactic radiosurgery for intracranial hemangiopericytomas. J Neurooncol 99: 115-122.

20. Ghia AJ, Chang EL, Allen PK, Mahajan A, Penas-Prado M, et al. (2013) Intracranial hemangiopericytoma: patterns of failure and the role of radiation therapy. Neurosurgery 73: 624-630.

21. Chamberlain MC, Glantz MJ (2008) Sequential salvage chemotherapy for recurrent intracranial hemangiopericytoma. Neurosurgery 63: 720-726.

22. Celik I, BaÅŸỸ Sil N, YalÃßin S, GÃ¹/4llÃ $1 / 4$ IH, Kars A, et al. (1997) Ifosfamide-based chemotherapy for recurrent or metastatic hemangiopericytoma. Acta Oncol 36: 348.

23. Pápai Z, Bodoky G, Szántó J, Poller I, Rahóty P, et al. (2000) The efficacy of a combination of etoposide, ifosfamide, and cisplatin in the treatment of patients with soft tissue sarcoma. Cancer 89: 177-180.

24. Chacko G, Chacko AG, Rajshekhar V, Muliyil JP (2006) Intracranial hemangiopericytomas: correlation of topoisomerase IIalpha expression with biologic behavior. Surg Neurol 65: 11-17.

25. Spatola C, Privitera G (2004) Recurrent intracranial hemangiopericytoma with extracranial and unusual multiple metastases: case report and review of the literature. Tumori 90: 265-268.

26. Jeong YI, Chang SE, Lee MW, Choi JH, Moon KC, et al. (2005) Case of cutaneous metastasis from intracranial hemangiopericytoma. Int J Dermatol 44: 870-872.

27. Jian BJ, Han SJ, Yang I, Waldron JS, Tihan T, et al. (2010) Surgical resection and adjuvant radiotherapy for a large pineal hemangiopericytoma. J Clin Neurosci 17: 1209-1211.

28. Ghia AJ, Allen PK, Mahajan A, Penas-Prado M, McCutcheon IE, et al. (2013) Intracranial hemangiopericytoma and the role of radiation therapy: a population based analysis. Neurosurgery 72: 203-209. 